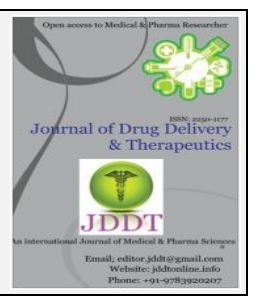

Open $\odot$ Access

Research Article

\title{
Effect of Cross Linking on Evaluation of Chitosan Coated Pellets of Glipizide
}

\author{
Nihar Ranjan Kar* and Kanhu Charan Pati \\ Gayatri Institute of Science and Technology, Gunupur, Dist-Rayagada, Odisha, India
}

\begin{abstract}
The objective of the current work is to develop and evaluate the effect of cross linking on drug release of chitosan pellets of Glipizide. Hence the present work was aimed to formulate glipizide pellets with a view to achieve and to maintain the plasma concentration for con siderable period by controlling the release so to decrease the occurrence of doses and also to recover the patient fulfillment. Here the pellets of glipizide are designed by Pan Coating technique with solution layering with and without cross linking by Gluterald ehyde effectively. Then the optimized formulations are aimed to study the effects of polymer and cross linking on different evaluation parameters including the drug release study. Finally the conclusion was to arrive at better formulation based on comparison amongst the studied ones.
\end{abstract}

Keywords- Pellets, Glipizide, Chitosan, Gluteraldehyde, Cross linking, In-Vitro characterization, Drug release study

Article Info: Received 12 July 2019; Review Completed 22 Aug 2019; Accepted 27 Aug 2019; Available online 30 Aug 2019

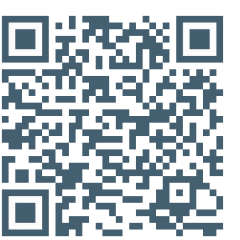

Cite this article as:

Kar NR, Pati KC, Effect of Cross Linking on Evaluation of Chitosan Coated Pellets of Glipizide, Journal of Drug Delivery and Therapeutics. 2019; 9(4-A):237-245 http://dx.doi.org/10.22270/jddt.v9i4-A.3416

*Address for Correspondence:

Nihar Ranjan Kar, Gayatri Institute of Science and Technology, Gunupur, Dist-Rayagada, Odisha, India. Email: nihar_795@rediffmail.com

\section{INTRODUCTION}

Pellets are used in decrease of dosage course of therapy and gastrointestinal annoyance, in addition controlling the drug discharge and escalating the absorption of the active component, moreover some of the useful properties of the pellet formulations are being excellent candidates for the release of the drug substances due to minimizing the dose clearance effect. The reproducibility of the discharge distinctiveness from pellet formulations is also a lot enhanced with respect to the single-unit dosage forms. They are proper system for film coating concerning the low surface area-volume ratios. Also, confrontation to peripheral factors such as moisture, air and light are the most valuable properties of these dosage forms ${ }^{1}$. Pellets have been extensively explored to build up controlled and delayed release oral formulations that release the active constituents in the gastrointestinal tract at prearranged rates. They are extensively accepted due to their straight action to objective tissue so as to get the most out of the drug delivery at the preferred site of action within the definite time period in the gastrointestinal tract. Pellets are less vulnerable to dose clearance and customized release profiles (pulsed, sustained or delayed) can be achieved by careful selection of polymers and method of preparation. Different chemically attuned as well as contrary pellets can be united collectively and conveyed in the gastrointestinal tract either at the similar sites or dissimilar sites. Scientifically, pellets are significantly spherical instinctively strong agglomerates of powder particles, organized by a specialized granulation process, ISSN: 2250-1177 known as pelletization, which was fashioned by the agglomeration of fine powdered adjuvants and drugs collectively that directs to the creation of small free flowing powders of spherical or semi spherical sizes².

\section{MATERIALS AND METHODS}

\section{Materials}

Materials and Methods: Glipizide was a gift sample from Sidmak Laboratories, Gujarat and all other chemicals/reagents used were of analytical grade and standard.

\section{Methods:}

\section{Preparation of drug solution layer:-}

Pan coating method was employed in order to set up spherical pellets. Glipizide 0.5 gm was integrated into nonpareils seeds by spurting glipizide in a solution in methylene chloride containing polyvinyl pyrolidone (PVP 30K) as a binder $10 \mathrm{gm}$ and talc $20 \mathrm{gm}$ as antiadherants. The declared amounts of glipizide and PVP K 30 were suspended in methylene chloride solution separately. After mixing each solution for a certain period of time, these two solutions were mixed together and necessary amount of methylene chloride is added up to the desired volume was made. The coating solution was sprayed over the non-pareils seed by using Laboratory size coating machine with the speed of 20rpm.The pressure of the spray gun was set at $4 \mathrm{~atm}$ and $1200 \mathrm{ml}$ coating solution was misted over $70 \mathrm{gm}$ of non 
pareil seeds . No heating was applied during the preparation for drying the pellets.The pellets were dehydrated at room temperature up to an invariable weight ${ }^{3}$.

Table 1: Composition of core pellets

\begin{tabular}{|l|l|}
\hline Ingredients & Quantity in gm \\
\hline Glipizide & 0.5 \\
\hline Polyvinylpyrollidone (PVP K 30 ) & 10.0 \\
\hline Talc Q.S. & 20.0 \\
\hline Methylene Chloride Q.S. & $300 \mathrm{ml}$ \\
\hline
\end{tabular}

Preparation of drug loaded pellet: (without Glutareldehyde)

The chitosan Solution was prepared by dissolving the stated amount of chitosan, i.e.1 gm (F1) and 1.25gm (F2) in 1\% acetic acid solution make volume upto $100 \mathrm{ml}$. Stirred for 30 mins by using stirrer. $100 \mathrm{gm}$ of drug loaded pellets were used for coating. The chitosan solution was sprayed radially using spray gun onto the rotating glipizide loaded pellets and very small amount of talc powder was sprayed onto the pellets intermittently to avoid the agglomeration of the pellets. The pellets were dried at $60^{\circ} \mathrm{c}$ temperature in vacuum oven up to $24 \mathrm{~h}$. Weight gain achieved was $10 \%$ $\mathrm{w} / \mathrm{w}$ and $20 \% \mathrm{w} / \mathrm{w}$ respectively 4 .

preparation of drug loaded pellet: (with Gluteraldehyde)

The chitosan Solution was prepared by dissolving the stated amount of chitosan, i.e.1 gm in 1\% acetic acid solution make volume upto $100 \mathrm{ml}$. Stirred for 30 mins by using a magnetic stirrer. $5 \mathrm{ml}$ of Gluteraldehyde ( 0.5 to $5 \%$ ) is added to the chitosan solution, and then this solution stirred for another $1 \mathrm{hr}$ at a magnetic stirrer then volume was made upto the mark and these solutions were kept for about 30 minutes.100 gm of drug loaded pellets were used for coating. The crosslinked chitosan solution of different \% of gluteraldehyde was sprayed radially using spray gun onto the rotating glipizide loaded pellets and very small amount of talc powder was sprayed onto the pellets intermittently to avoid the agglomeration of the pellets. The pellets were dried at $60^{\circ} \mathrm{c}$ temperature in vacuum oven up to $24 \mathrm{~h}{ }^{4}$. The pellets obtained are named as $\mathrm{F} 3(0.5 \%), \mathrm{F} 4(1.0 \%)$, $\mathrm{F} 5(2.0 \%), \mathrm{F} 6(3.0 \%), \mathrm{F} 7(4.0 \%)$ and $\mathrm{F} 8(5.0 \%)$ according to the conc ${ }^{\mathrm{n}}$ of Gluteraldehyde taken.

Table 2: Composition of coating solution for core pellets

\begin{tabular}{|l|l|l|l|l|l|l|l|l|}
\hline Ingredients & F1 & F2 & F3 & F4 & F5 & F6 & F7 & F8 \\
\hline $\begin{array}{l}\text { Glipizide } \\
\text { (pellets) }\end{array}$ & $100 \mathrm{gm}$ & $100 \mathrm{gm}$ & $100 \mathrm{gm}$ & $100 \mathrm{gm}$ & $100 \mathrm{gm}$ & $100 \mathrm{gm}$ & $100 \mathrm{gm}$ & $100 \mathrm{gm}$ \\
\hline Chitosan & $1.0 \mathrm{mg}$ & $1.25 \mathrm{gm}$ & $1.0 \mathrm{gm}$ & $1.0 \mathrm{gm}$ & $1.0 \mathrm{gm}$ & $1.0 \mathrm{gm}$ & $1.0 \mathrm{gm}$ & $1.0 \mathrm{gm}$ \\
\hline $\begin{array}{l}\text { Acetic Acid } \\
\text { Solution(\%v/v) }\end{array}$ & 1.0 & 1.0 & 1.0 & 1.0 & 1.0 & 1.0 & 1.0 & 1.0 \\
\hline Gluteraldehyde(\%) & ----- & ----- & 0.5 & 1.0 & 2.0 & 3.0 & 4.0 & 5.0 \\
\hline Talc & q.s. & q.s. & q.s. & q.s. & q.s. & q.s. & q.s. & q.s. \\
\hline Water & q.s. & q.s. & q.s. & q.s. & q.s. & q.s. & q.s. & q.s. \\
\hline
\end{tabular}

Evaluation of pellets 5 : The pellets were portrayed by their micromeritic possessions, such as, flow properties, bulk density, tapped density, and Hauser ratio.

\section{Angle of repose:-}

Angle of repose $\mathrm{q}$ of the pellets, which measures the resistance to particle flow, was determined by a fixed funnel method and calculated as Tan $\mathrm{q}=2 \mathrm{H} / \mathrm{D}$, Where $\mathrm{H}$ is the height of the pellets heap and $\mathrm{D}$ is diameter of the spherical surface of the pellets heap that is formed on a graph paper after making the pellets flow from the glass funnel.

Bulk Density:-Apparent bulk density was found out by introducing some fixed weight of pellets into graduated cylinder and calculating the volume. Bulk Volume will be estimated by tapping the graduated cylinder in bulk density apparatus up to a fixed volume obtained. The final volume is known as bulk volume. Bulk density was calculated by the formula,

Bulk density $(\mathrm{g} / \mathrm{ml})=$ Weight of sample in grams / Bulk volume.

Tapped Density-The tapping method was applied to find out the tapped density as follows, Tapped density = Weight of sample / Volume of pellets after tapping 50 times
Hauser ratio- It gives a suggestion of the degree of densification which could effect from vibration of the feed hopper.

Hauser ratio= Tapped density/ Fluff density.

Lower Hausner's ratio = Better flowability, Higher Hausner's ratio = poor flowability

Friability - The test of Friability was carried out by using 20 pellets (Roche friabilator). Friability of the crosslinked chitosan coated pellets as characterization parameters were evaluated.

Drug content $100 \mathrm{mg}$ of glipizide loaded pellets were triturated to get fine powder, and then it was transferred into $100 \mathrm{ml}$ volumetric flask and dissolved in $100 \mathrm{ml}$ phosphate buffer at $\mathrm{pH}$ 7.4. The resulting mixture was stirred up on an orbital shaker for $24 \mathrm{~h}$. Quantity of samples equivalent to $10 \mathrm{mg}$ of glipizide were taken, filtered through $0.45 \mu \mathrm{m}$ whatman filter paper, diluted suitably and analysed at $276 \mathrm{~nm}$ using spectrophotometer. The assay was performed and mean values were taken.

\section{Scanning electron microscopy (SEM)}

The morphology of pellets were studied by scanning electron microscopy (SEM), which was performed to CODEN (USA): JDDTAO 
characterize the surface of formed pellets. SEM photographs of the chitosan coated nonpareil pellets of glipizide were taken before and after coating. The coated pellets were scanned and the micrographs were examined.

\section{In vitro Drug release studies}

A USP basket apparatus has been used to study in-vitro drug release from pellets.

The dissolution test for coated pellets equivalent to $10 \mathrm{mg}$ was performed in using dissolution test apparatus. The dissolution test was performed using $900 \mathrm{ml} \mathrm{pH} 7.4$ buffer, at $37 \pm 0.5^{\circ} \mathrm{C}$ and $100 \mathrm{rpm}$. The volume was replenished with the same amount of fresh dissolution fluid each time to maintain the sink condition. Withdrawn samples $(10 \mathrm{~mL})$ were analyzed spectrophotometrically at $276 \mathrm{~nm}$. Then the cumulative percentage drug release was calculated.

\section{Stability studies of the optimized formulation}

The stability studies were carried out according to ICH and WHO guidelines to assess the drug and formulation stability. Best found formulation that is F8 was sealed in aluminum packaging having a polyethylene coating on the inside.

Samples were kept in a humidity chamber maintained at $40^{\circ} \mathrm{C}$ and $75 \% \mathrm{RH}$ for 3 months .At the end of studies, samples were analyzed for the drug content, in vitro release profile and other physicochemical parameters.

\section{RESULT AND DISCUSSION}

\section{Characterization of Glipizide:}

Melting point determination: The melting point of Glipizide was determined by Melting point apparatus using capillary method. It was found to be $208^{\circ} \mathrm{C}$ which complies with reported value 6 .

\section{Evaluation of Chitosan Coated Pellet (Non Cross Linked with Gluteraldehyde)}

\section{Micromeritic properties:}

The effect of polymer concentration on the micromeritic properties of pellets was studied. The mean particle size of pellets increased with an increasing polymer concentration. This may be due to increased total solids onto the pellets, which resulted in uniform coating may lead to uniform particle size distribution. Coating of the non pareils did not have significant effect on flow properties of the pellets as shown in Table -1 because non pareils possess definite structure and mechanical strength ${ }^{7}$.

Table 3: Micromeritic properties of chitosan coated glipizide loaded pellets

\begin{tabular}{|c|c|c|c|c|c|}
\hline Formulation & $\begin{array}{c}\text { Bulk density } \\
\text { (gm/ml) }\end{array}$ & $\begin{array}{c}\text { Tapped density } \\
\text { (gm/ml) }\end{array}$ & $\begin{array}{c}\text { Angle of } \\
\text { repose }^{\mathbf{a}}\end{array}$ & $\begin{array}{c}\text { Hausner } \\
\text { ratio }^{\mathbf{a}}\end{array}$ & $\begin{array}{c}\text { Loss on } \\
\text { dryinga (\%) }^{\text {(\%) }}\end{array}$ \\
\hline $\mathrm{F} 1$ & $0.714 \pm 0.11$ & $0.725 \pm 0.03$ & $15.01 \pm 0.02$ & $1.013 \pm 0.23$ & $3.08 \pm 0.27$ \\
\hline $\mathrm{F} 2$ & $0.723 \pm 0.02$ & $0.735 \pm 0.01$ & $16.61 \pm 0.30$ & $1.014 \pm 0.30$ & $3.51 \pm 0.35$ \\
\hline
\end{tabular}

a Mean $\pm \mathrm{SD} ; \mathrm{n}=3$.

Angle of repose of pellet formulation was found to be 15.01 and 16.61 for F1 and F2 respectively which shows that pellets exhibit excellent flow properties. Hausner ratio closer to 1 indicates good flow property and packing ability.

\section{Friability Test of the formulations:}

Results of friability test for chitosan coated pellets were found out be $0.03 \%$ for F1 and $0.04 \%$ for F2. Pellet formulations F1 and F2 achieved friability values less than $1 \%$ as per USP limit for tablets. Thus all pellets passed the USP friability test.

\section{Drug Content:}

Drug content of chitosan coated non pareils was found out to be $98.00 \%$ for $\mathrm{F} 1$ and $97.25 \%$ for F2. So the drug content was found out be within the limit.

\section{In vitro Drug release studies:}

\section{Spectrophotometer Method Development for Estimation of Glipizide in Bulk:}

Selection of wavelength for analysis and preparation of standard calibration curve in Phosphate buffer $\mathrm{pH}$ (7.4)

\section{Selection of Analytical wavelength:}

\section{UV- spectrum:}

The spectrum of glipizide showed considerable linearity at wavelength $276 \mathrm{~nm}$ in phosphate buffer ( $\mathrm{pH}$ 7.4). This wavelength was used for determination of drug content of formulation, in vitro drug dissolution studies.

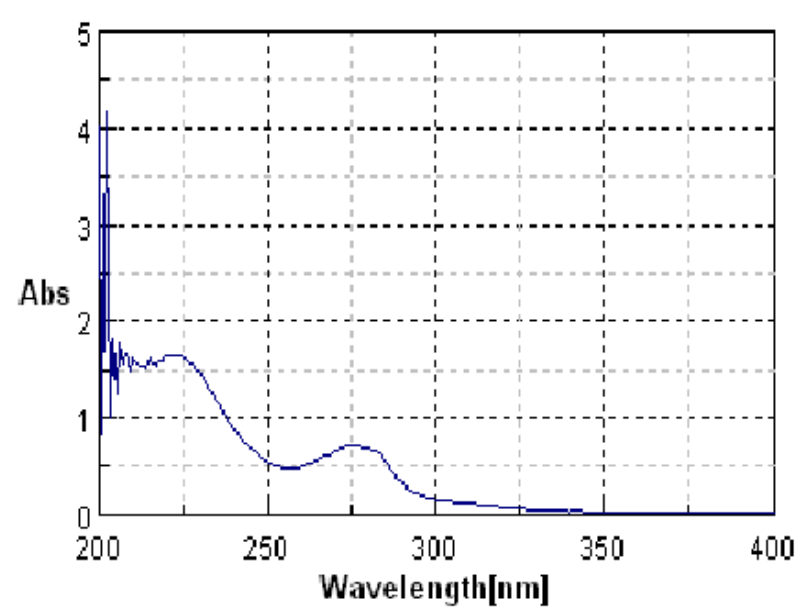

Figure 1: UV spectrum of Glipizide.

\section{Standard Curve ${ }^{8}$ :}

Calibration curve was prepared by taking absorption of diluted stock solutions at wavelength $276 \mathrm{~nm}$. Beer's law was obeyed in the concentration range $5-50 \mu \mathrm{g} / \mathrm{ml}$. 
Table 4: Standard curve of Glipizide in phosphate buffer (pH 7.4)

\begin{tabular}{|c|c|c|}
\hline Sr.No. & $\begin{array}{c}\text { Concentration } \\
(\boldsymbol{\mu g} / \mathbf{m l})\end{array}$ & Absorbance \\
\hline 1 & 5 & 0.1449 \\
\hline 2 & 10 & 0.2548 \\
\hline 3 & 15 & 0.3995 \\
\hline 4 & 20 & 0.5441 \\
\hline 5 & 25 & 0.6487 \\
\hline 6 & 30 & 0.7761 \\
\hline 7 & 35 & 0.9082 \\
\hline 8 & 40 & 1.0225 \\
\hline 9 & 45 & 1.1574 \\
\hline 10 & 50 & 1.2756 \\
\hline
\end{tabular}

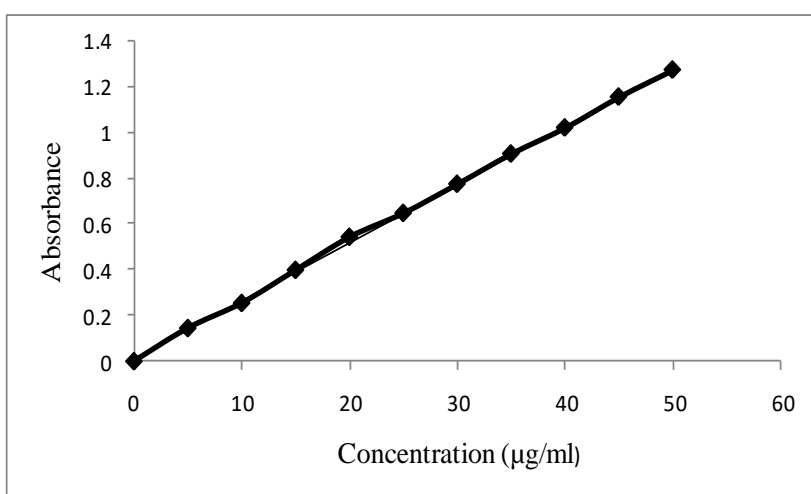

Figure 2: Calibration Curve of Glipizide in phosphate buffer pH 7.4

The standard curves with equations for regression line and $\mathrm{R}^{2}$ values are given below. Standard curve with phosphate buffer ( $\mathrm{pH}$ 7.4) was used for drug content evaluation and for dissolution studies.

$\mathrm{Y}=0.0252 \mathrm{x}+0.0189\left(\mathrm{R}^{2}=0.9994\right)$
In vitro dissolution studies of glipizide from different pellets were performed in phosphate buffer ( $\mathrm{pH}$ 7.4) using USP Type I dissolution test apparatus. In vitro release experiments were evaluated in order to investigate the effect of chitosan polymer and the amount of the polymer content on the release pattern of pellets loaded with glipizide.Chitosan has high water-solubility and swelling properties in aqueous media. Percentage release of chitosan coated formulations (F1 and F2) was shown in Table 3.

Table 5: Dissolution study of chitosan coated glipizide loaded pellets

\begin{tabular}{|c|c|c|}
\hline Time (Hrs) & \multicolumn{2}{|c|}{ Cumulative \% Drug Release } \\
\cline { 2 - 3 } & F1 & F2 \\
\hline 1 & 48.98 & 40.36 \\
\hline 2 & 52.28 & 46.21 \\
\hline 3 & 60.23 & 55.25 \\
\hline 4 & 68.45 & 63.06 \\
\hline 5 & 79.12 & 70.28 \\
\hline 6 & 84.23 & 77.87 \\
\hline 7 & 89.86 & 82.41 \\
\hline 8 & 96.25 & 93.87 \\
\hline
\end{tabular}

a Mean \pm SD; $n=3$.

Dissolution profile of chitosan coated pellets showed that the burst release of drug from the pellets it might due to the high aqueous solubility of chitosan. The basic molecular parameters of chitosan, like molecular weight and degree of deacetylation, influence the drug release. Chitosan is hydrophilic, retains water in its structure and forming gels spontaneously6. In the present study chitosan used was of $86 \%$ deacylated. The chitosan with high degree of deacylation showed a low degree of swelling 7 , hence formulation showed the burst release, and also the poor gel formation of chitosan in phosphate buffer pH 7.4 also contributed the fact ${ }^{8}$. As the coating thickness increased onto the glipizide pellets the release was slightly decreased. Formulation F1 and F2 showed the 96.25\% and 93.87\% release in $8 \mathrm{~h}$ which is shown in Fig.03.

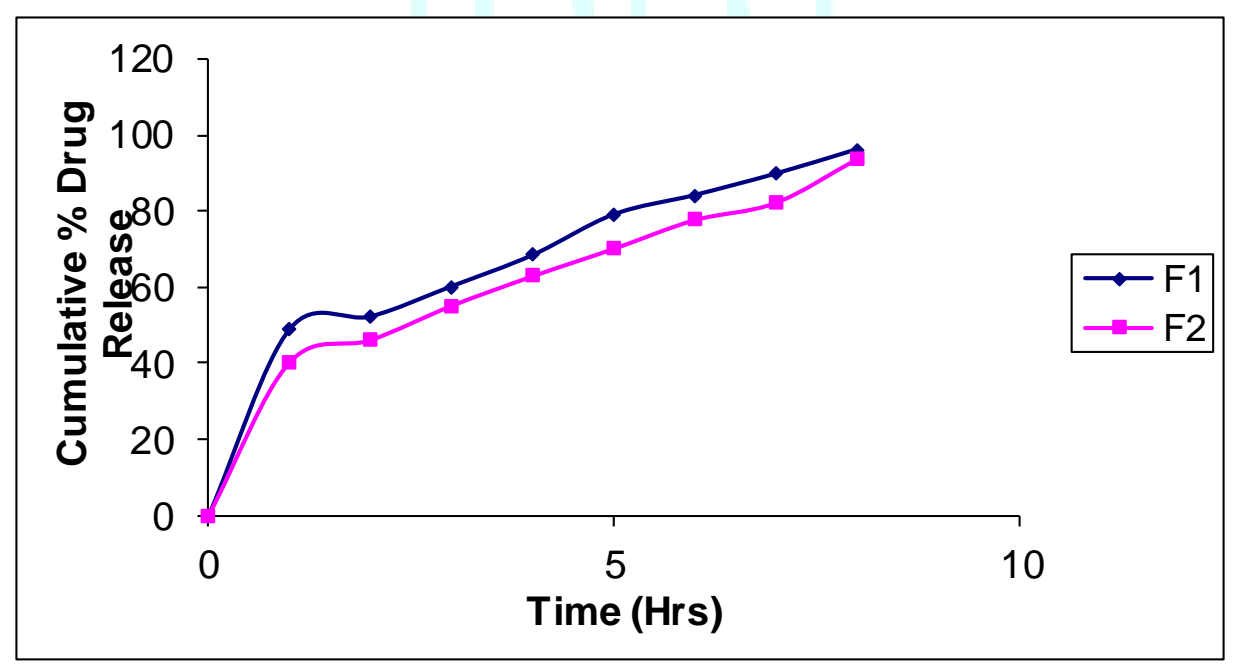

Figure 3: Dissolution profile of chitosan coated glipizide loaded pellets

Drug release models ${ }^{9}$ :

It was found that Formulations F1 and F2 showed $96.25 \%$ and $93.87 \%$ in 8 hours. The value of $\mathrm{n}$ was found to 0.34 and 0.403 for F1 and F2 respectively, which indicate that formulations followed Korsemeyer-Peppas model for drug release. 
6.6.1.1.6. Morphology of coated non pareils (SEM):

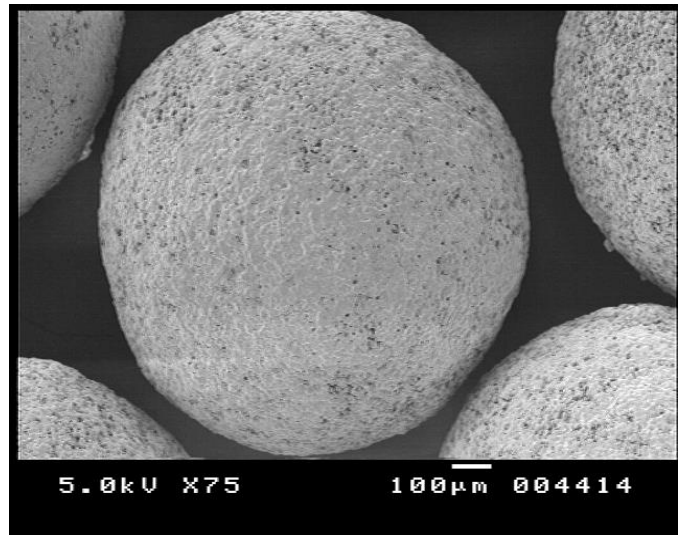

[A]

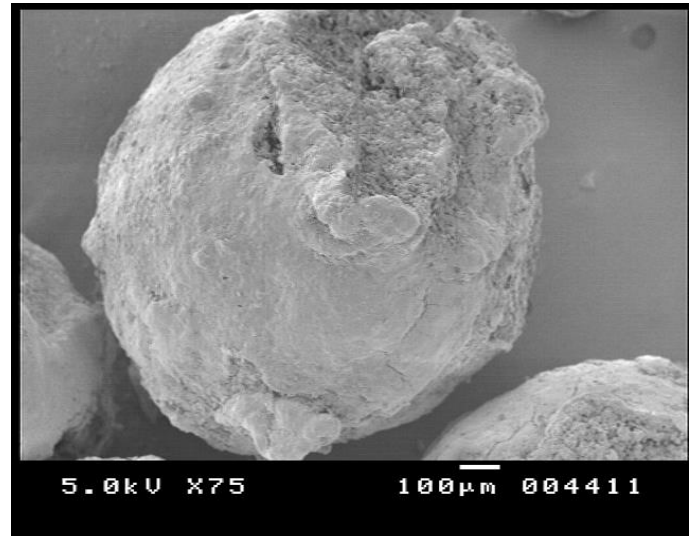

[B]

Figure 4: SEM Photograph of Chitosan coated pellets

A - Non pareils. B - Chitosan coated pellets.

The view of the non pareils showed a smooth surface whereas coated pellets showed the rough /porous surface structure is caused due to rapid loss of moisture from the wet mass with a high liquid content. From the SEM data it was clear that chitosan pellets showed the burst release, because of the pellet get eroded.

Evaluation of Cross linked Chitosan Pellets (with Gluteraldehyde)

\section{Micromeritic properties: ${ }^{10}$}

Coating of the non pareils did not have significant effect on flow properties of the pellets as shown in Table 24 because non pareils possess defined structure and mechanical strength. The angle of repose of pellets ranged from $13.84 \pm 0.2$ to $17.12 \pm 0.1$. The values of angle of repose and Hausner ratio indicate excellent flowing properties. Micromeritic properties are mentioned in Table 05.

Table 6: Micromeritic properties of cross-linked chitosan coated glipizide loaded pellets

\begin{tabular}{|c|c|c|c|c|c|}
\hline Formulation & $\begin{array}{c}\text { Bulk density } \\
\text { (gm/ml) }\end{array}$ & $\begin{array}{c}\text { Tapped density } \\
\text { (gm/ml) }\end{array}$ & $\begin{array}{c}\text { Angle of } \\
\text { repose }^{\mathbf{g}}\end{array}$ & $\begin{array}{c}\text { Hausner } \\
\text { ratioa }^{\mathbf{a}}\end{array}$ & $\begin{array}{c}\text { Loss on } \\
\text { dryinga }^{\mathbf{( \% )}}\end{array}$ \\
\hline F3 & $0.8523 \pm 0.03$ & $0.923 \pm 0.02$ & $14.31 \pm 0.02$ & $1.083 \pm 0.01$ & $3.08 \pm 0.3$ \\
\hline F4 & $0.8142 \pm 0.04$ & $0.825 \pm 0.28$ & $15.12 \pm 0.1$ & $1.014 \pm 0.02$ & $2.98 \pm 0.18$ \\
\hline F5 & $0.8232 \pm 0.1$ & $0.835 \pm 0.4$ & $13.84 \pm 0.2$ & $1.014 \pm 0.1$ & $3.24 \pm 0.2$ \\
\hline F6 & $0.8146 \pm 0.02$ & $0.822 \pm 0.04$ & $16.52 \pm 0.01$ & $1.0095 \pm 0.3$ & $3.10 \pm 0.32$ \\
\hline F7 & $0.8525 \pm 0.04$ & $0.893 \pm 0.01$ & $17.12 \pm 0.1$ & $1.047 \pm 0.04$ & $3.45 \pm 0.2$ \\
\hline F8 & $0.8120 \pm 0.11$ & $0.867 \pm 0.3$ & $16.71 \pm 0.15$ & $1.067 \pm 0.03$ & $3.34 \pm 0.4$ \\
\hline
\end{tabular}

F: Formulations a Mean \pm SD; $\mathrm{n}=3$.

\section{Friability of the formulations ${ }^{11}$ :}

Friability of the crosslinked chitosan coated pellets as characterization parameters were evaluated and the results in the range of 0.02 to $0.20 \%$. All pellet formulations achieved friability values less than $0.4 \%$. Thus all pellets passed the USP friability test. Values of friability test are mentioned in Table 06.

Table 7: Friability of crosslinked chitosan coated glipizide loaded pellets

\begin{tabular}{|c|c|}
\hline Formulation & Friabilitya(\%) \\
\hline F3 & 0.20 \\
\hline F4 & 0.05 \\
\hline F5 & 0.10 \\
\hline F6 & 0.04 \\
\hline F7 & 0.02 \\
\hline F8 & 0.03 \\
\hline \multicolumn{2}{|c|}{ a Mean \pm SD; $=3}$. \\
\hline
\end{tabular}

Drug Content: 12

Drug content of crosslinked chitosan coated non pareils was found out to be in the range of $94.63 \%$ to $98.60 \%$ for formulation F3 to F8.So the drug content was found out be within the limit which shown in Table 07.

Table 8: Drug content of crosslinked chitosan coated glipizide loaded pellets

\begin{tabular}{|c|c|}
\hline Formulation & Drug Content $^{\mathbf{a}}$ (\%) \\
\hline F3 & 96.30 \\
\hline F4 & 94.63 \\
\hline F5 & 98.00 \\
\hline F6 & 95.12 \\
\hline F7 & 98.60 \\
\hline F8 & 97.25 \\
\hline \multicolumn{2}{|c|}{ a Mean \pm SD; $\mathrm{n}=3}$.
\end{tabular}

\section{In vitro Drug release studies: ${ }^{13}$}

In vitro dissolution studies of glipizide from different pellets were performed in phosphate buffer $(\mathrm{pH}$ 7.4) using USP Type I dissolution test apparatus. The in vitro release experiments were evaluated in order to investigate the effect of the crosslinker and the polymer on the drug release from the pellet formulations. Chitosan exhibits $\mathrm{pH}$-dependant 
swelling and controlled drug release properties. The chitosan is hydrophilic in nature in acidic media due to the presence of the amino group, but hydrophilicity and solubility of chitosan is changed on covalent crosslinking with aldehydes. However, the variations in hydrophilicity depend on the type of crosslinker and chitosan used for crosslinking. The cross-linking density has a remarkable effect on the release of drugs from the chitosan matrix systems. Higher densities tend to slow drug release more than lower densities. Crosslinking with gluteraldehyde increase the hydrophobic character in chitosan. It was observed that increase in concentration of gluteraldehyde, the release of the glipizide from the pellets is retarded and vieversa $^{9}$. Formulation F3, F4, F5 and F6 showed the glipizide release $95.02 \%, 95.93 \%, 92.93 \%$ and $92.20 \%$ in 9 , 10 and $11 \mathrm{~h}$. respectively which is shown in Table 27 . From the dissolution profile it is understood that as the percentage of gluteraldehyde increases the drug release was retarded. Formulation F7 and f8 showed the drug release $90.86 \%$ and $88.73 \%$ in 12 h., although the formulation F7 and F8 showed drug release similar but the formulation F8 showed the initial release less as compared to the formulation F7.

Table 09: Dissolution study of cross linked chitosan coated glipizide loaded pellets

\begin{tabular}{|c|c|c|c|c|c|c|}
\hline \multirow{2}{*}{$\begin{array}{c}\text { Time } \\
\text { (Hrs) }\end{array}$} & \multicolumn{7}{|c|}{ Cumulative \% drug release } \\
\cline { 2 - 7 } & F3 & F4 & F5 & F6 & F7 & F8 \\
\hline 1 & 39.28 & 32.63 & 28.33 & 22.75 & 18.04 & 12.22 \\
\hline 2 & 51.68 & 42.55 & 40.76 & 36.49 & 25.02 & 16.5 \\
\hline 3 & 58.63 & 51.68 & 49.43 & 44.49 & 30.70 & 21.98 \\
\hline 4 & 66.05 & 59.85 & 58.56 & 50.74 & 36.26 & 25.75 \\
\hline 5 & 72.88 & 67.60 & 62.36 & 58.43 & 41.68 & 38.33 \\
\hline 6 & 80.67 & 75.23 & 68.34 & 64.48 & 51.97 & 46.14 \\
\hline 7 & 85.02 & 85.64 & 73.06 & 69.33 & 61.31 & 55.17 \\
\hline 8 & 88.24 & 87.89 & 79.59 & 72.28 & 66.30 & 63.08 \\
\hline 9 & 95.02 & 93.29 & 84.34 & 80.79 & 75.54 & 69.33 \\
\hline 10 & - & 95.93 & 87.23 & 84.41 & 80.88 & 78.81 \\
\hline 11 & - & - & 92.93 & 92.2 & 85.56 & 84.16 \\
\hline 12 & - & - & - & - & 90.86 & 88.73 \\
\hline
\end{tabular}

a Mean \pm SD; $n=3$.

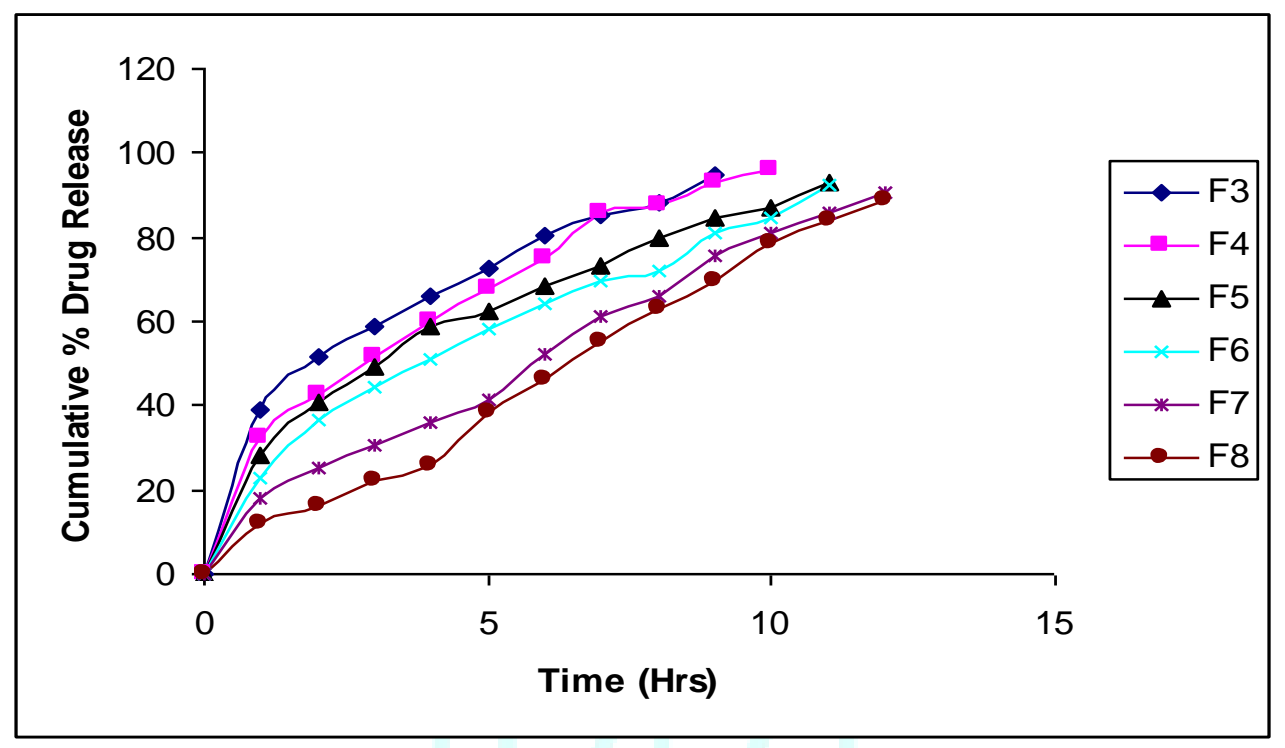

Figure 04: Dissolution profile of crosslinked chitosan coated glipizide loaded pellets

\section{Drug release models: ${ }^{14}$}

In vitro dissolution studies of glipizide pellets coated with crosslinked chitosan were performed in phosphate buffer (pH 7.4) for $12 \mathrm{~h}$ using USP Type I dissolution test apparatus. In case of controlled or sustained release formulations, diffusion, swelling and erosion are the three most important rate controlling mechanisms. Formulations containing swelling polymers showed swelling as well as diffusion mechanism, because in addition to diffusion, processes includes relaxation of polymer chains, imbibitions of water causing polymer to swell and changing them from initial glassy to rubbery state. Peppas described the concept drug release mechanism, values of $n$ i.e. release exponent for formulations are given in Table, value of $n$ as 0.5 indicates diffusion-controlled drug release and for the value 1.0 it indicates swelling-controlled drug release. Value of $n$ between 0.5 and 1.0 can be regarded as an indicator for both the phenomenon (anomalous transport). the value of $n$ from 1.0 to1.4 (i.e. more than 1.0) which indicates the release mechanism following anomalous transport, Case II transport and super case -II transport, respectively. Formulation F3 to F6 followed the peppas model and formulation $\mathrm{F} 4$ followed the matrix model, which describes the drug release as a diffusion process. Formulation F8 followed the zero order kinetics which is shown Table 09.

Table 10: Results of Kinetic treatment of Glipizide from pellet formulations (F3-F8)

\begin{tabular}{|c|c|c|c|c|}
\hline F & Best Fit Model & R & $\mathbf{n}$ & $\mathbf{k}$ \\
\hline F3 & Peppas & 0.9983 & 0.3995 & 38.7733 \\
\hline F4 & Matrix & 0.9989 & 0.2960 & 30.6638 \\
\hline F5 & Peppas & 0.9997 & 0.4880 & 28.6655 \\
\hline F6 & Peppas & 0.9981 & 0.5585 & 23.6099 \\
\hline F7 & Peppas & 0.9884 & 0.6903 & 15.6410 \\
\hline F8 & Zero & 0.9967 & 1.010 & 9.6882 \\
\hline
\end{tabular}

R- Regression coefficient, $\mathrm{n}$ - Release exponent and kProportionality constant 
Morphology of coated non pareils (SEM): 15

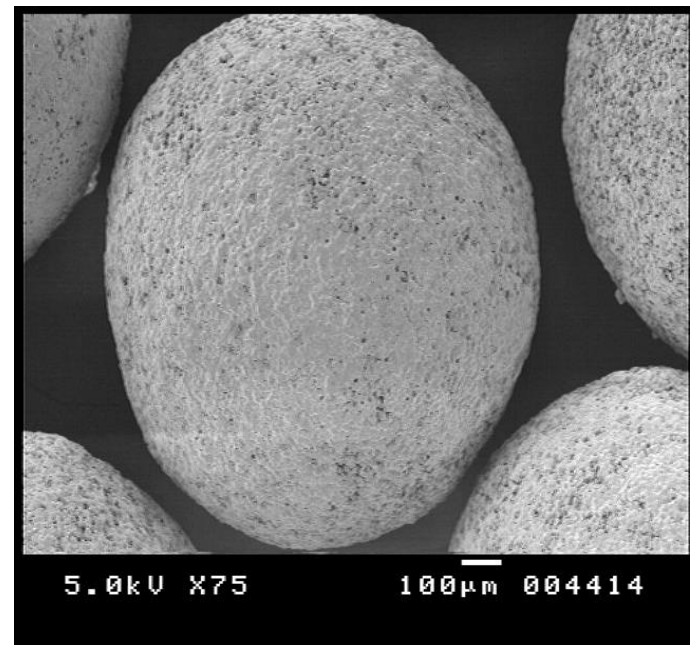

[C]

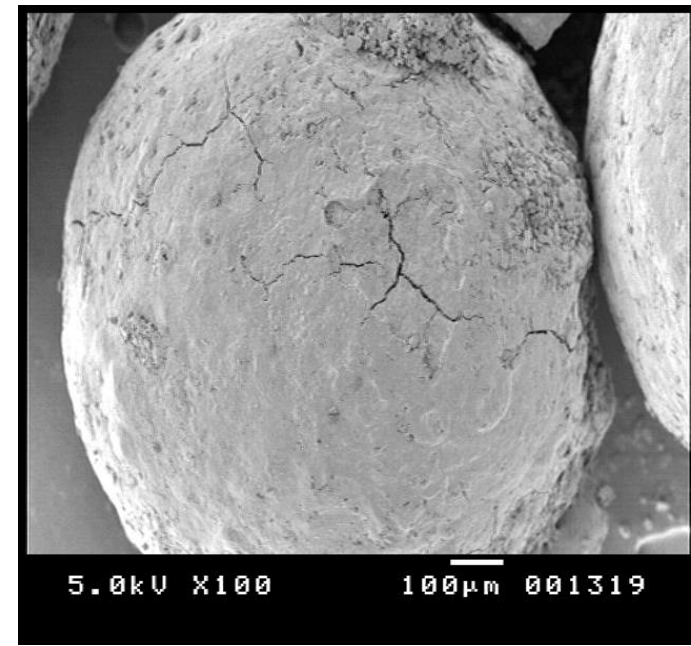

[D]

Figure 5: SEM Photograph of crosslinked chitosan coated pellets

C - Non Pareils. D - Crosslinked chitosan coated.

SEM of the crosslinked chitosan surface having the less cracks and surface of pellets more compact than the chitosan coated pellets, hence it can be correlated that dissolution profile of the chitosan and crosslinked coated pellets which is shown in Fig. 05

\section{Drug-Excipient Compatibility-16}

IR Spectrum Interpretation: The IR spectrum of Glipizide is shown in Fig.06

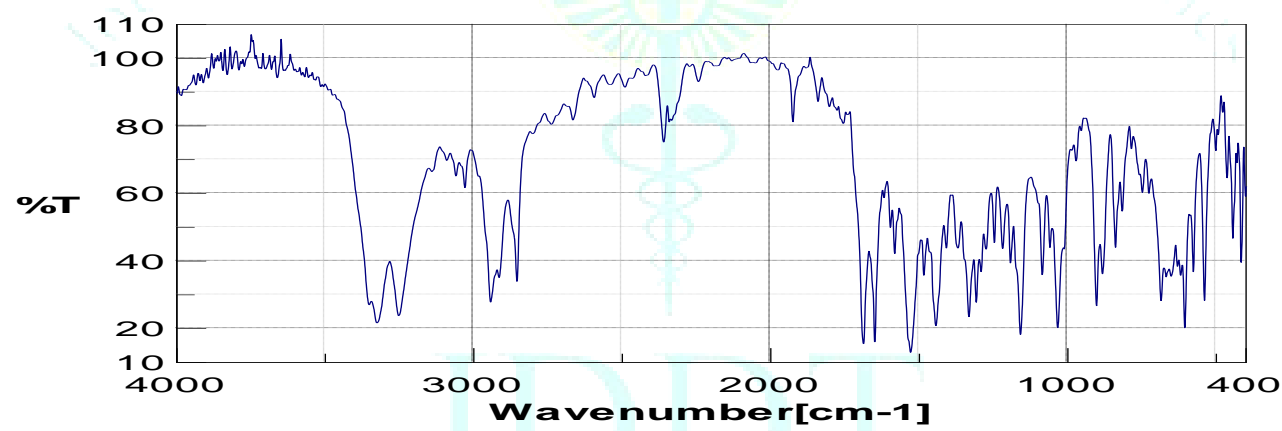

Figure-06 IR spectrum interpretation of Glipizide pure drug

Table 11: IR spectrum interpretation of Glipizide pure drug

\begin{tabular}{|l|l|}
\hline Wave number(cm-1) & Assignment \\
\hline 3354 & $\mathrm{~N}-\mathrm{H}$ stretching of $\mathrm{NH}_{2}$ \\
\hline 2943 & $\mathrm{C}-\mathrm{H}_{2}$ Aliphatic group \\
\hline 1689 & $\mathrm{C}=$ O stretching \\
\hline 1651 & $\mathrm{C}=$ N Aliphatic group \\
\hline 1529 & $\mathrm{C}-\mathrm{H}$ stretching \\
\hline
\end{tabular}

Interpretation of Covalent bonding in IR $\operatorname{spectrum}\left(\mathrm{cm}^{-1}\right)$

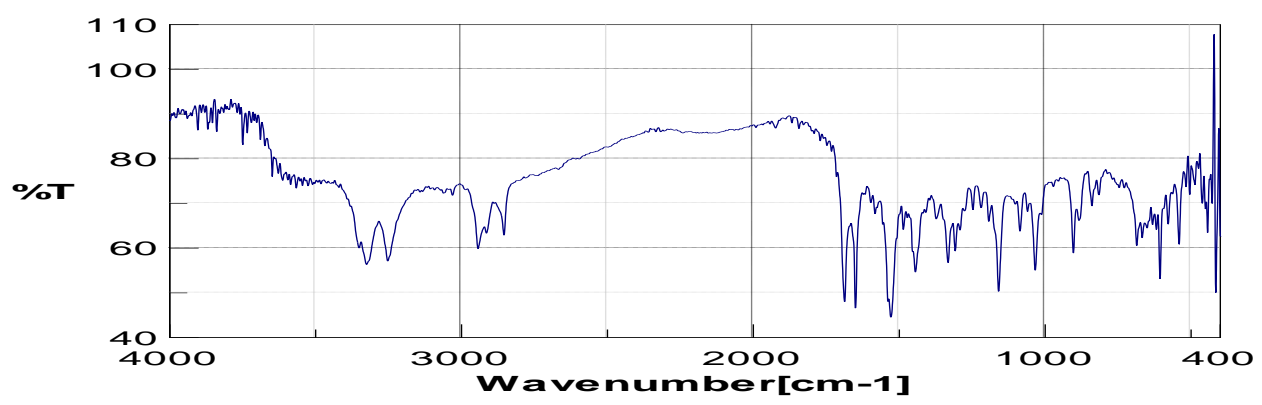

Figure-07-IR Spectra of Glipizide-Chitosan (non cross linked) 


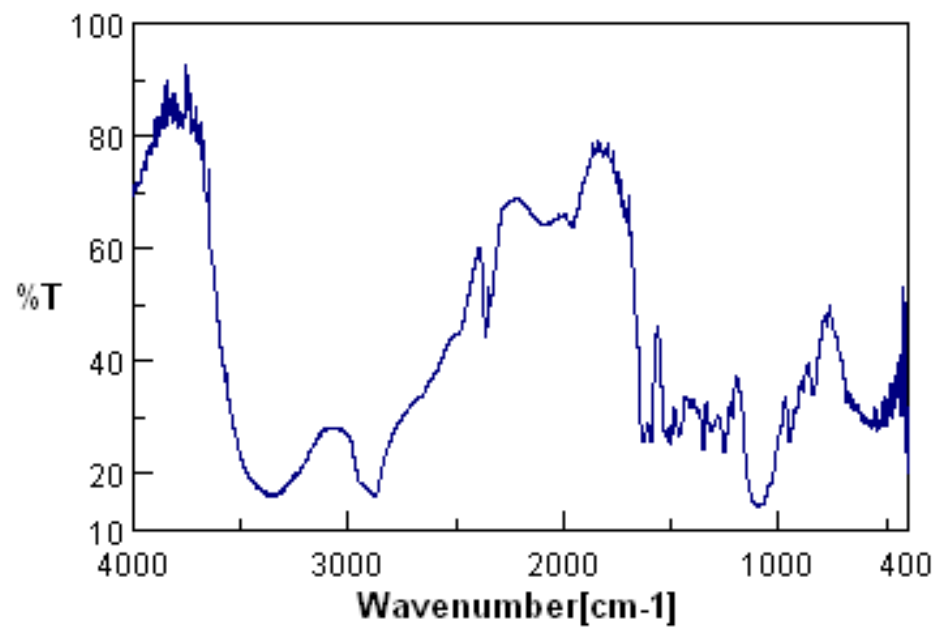

Figure 08: IR Spectra of Glipizide-chitosan cross linked with Gluteraldehyde

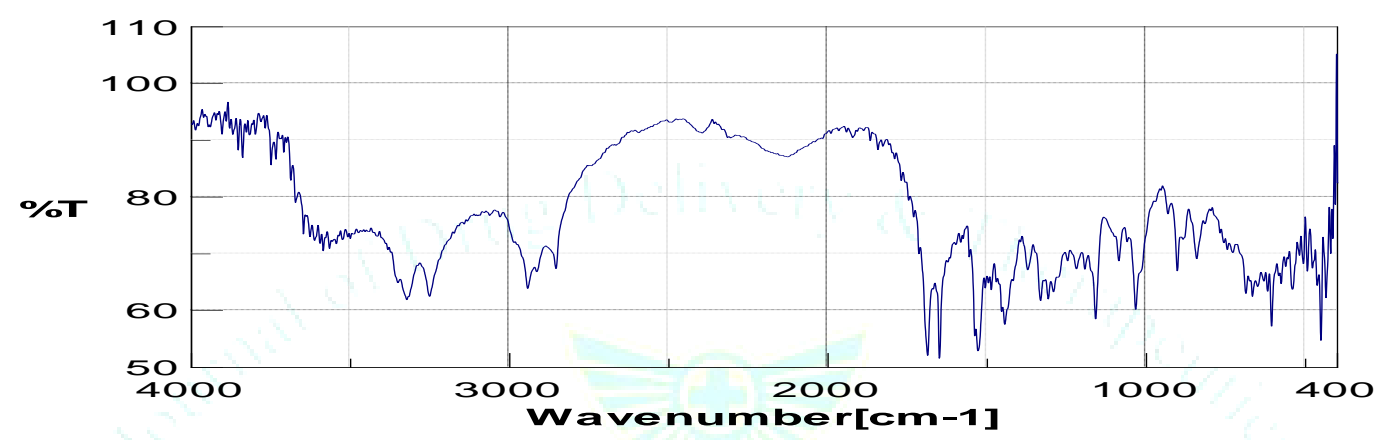

Figure-09-IR spectrum Analysis of PVP K 30 + Drug

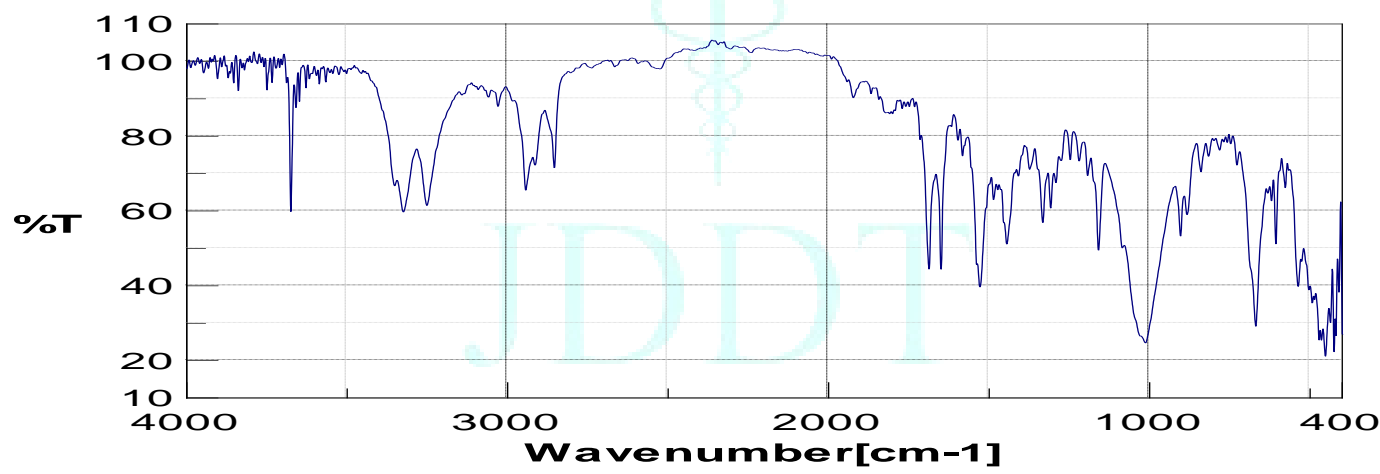

Figure-10-IR spectrum Interpretation of Talc + Drug

IR spectra of various glipizide-chitosan mixture(non crosslinked and cross linked) showed no interaction with the drug and prominent peaks of the drug were not affected. Thus, no interaction was observed between the drug and excipients. Hence drug excipients compatibility was established. There was neither shift and nor disappearance of characteristic peaks suggesting that there is no interaction between glipizide and other excipients or no degradation in drug molecule.

\section{Stability studies: 17}

The stability studies were carried out on optimized formulation. The samples were stored at $40{ }^{\circ} \mathrm{C} \pm 2{ }^{\circ} \mathrm{C} / 75 \% \pm$ $5 \% \mathrm{RH}$ for three months to access their stability. The protocol of stability studies were in compliance with WHO guidelines for stability testing intended for the global market. After 30 days samples were withdrawn and retested for drug content and drug release studies. The best found formulations F8, F11 and F14 did not show any significant change in drug content when kept at different condition and periods. No significant difference in values of $\%$ drug release after $12 \mathrm{~h}$ observed during the stability studies. It indicates that irrespective of concentration of polymer, this formulation was able to retain its stability. 
Table 12: Stability studies of selected formulations

\begin{tabular}{|c|c|c|c|c|c|c|}
\hline \multirow{2}{*}{ Time period } & \multicolumn{3}{|c|}{ Drug content (\%) } & \multicolumn{3}{|c|}{ In vitro drug release after $12 \mathrm{hr}(\%)$} \\
\hline & F2 & F7 & F8 & F2 & F7 & F8 \\
\hline Initial & 97.25 & 98.60 & 97.25 & 93.87 & 90.86 & 88.73 \\
\hline \multicolumn{7}{|c|}{ One month } \\
\hline Ambient & 96.35 & 96.53 & 96.86 & 93.67 & 90.34 & 88.51 \\
\hline $40^{\circ} \mathrm{c} / 75 \% \mathrm{RH}$ & 96.01 & 95.48 & 96.39 & 93.23 & 90.13 & 88.12 \\
\hline \multicolumn{7}{|c|}{ Two month } \\
\hline Ambient & 96.16 & 96.11 & 96.24 & 92.89 & 89.91 & 88.38 \\
\hline $40^{\circ} \mathrm{c} / 75 \% \mathrm{RH}$ & 95.11 & 94.23 & 96.36 & 92.72 & 90.01 & 94.02 \\
\hline \multicolumn{7}{|c|}{ Three month } \\
\hline Ambient & 95.89 & 96.01 & 96.16 & 84.01 & 88.23 & 93.51 \\
\hline $40^{\circ} \mathrm{c} / 75 \% \mathrm{RH}$ & 93.76 & 93.65 & 95.10 & 82.78 & 86.89 & 92.78 \\
\hline
\end{tabular}

\section{SUMMARY AND CONCLUSION}

Glipizide is an agent belonging to sulfonylurea group used for the treatment of non-insulin dependent diabetes mellitus (NIDDM). Glipizide was administered once or twice in a day as tablet dosage form in dose regimen of 5 to $20 \mathrm{mg}$. The purpose of present work was to prepare sustained release pellets of glipizide in order to decrease the dosage regimen and to maintain the necessary blood concentration. Step by step studies were carried out to develop formulation of pellets using solution layering method. Non pareils (inert materials) were loaded with drug solution. To achieve sustained release of drug from the pellets, they were coated with polymer like chitosan and further cross linked with Gluteraldehyde. The differentnevaluation parameter was conducted among different formulations for knowing the effect of cross linking on the release study of glipizide. Pellets were evaluated for micromeritic properties as well as for in - vitro drug release in $\mathrm{pH} 7.4$ phosphate buffer. The best found formulations were subjected to the stability studies.

\section{Following conclusions may be drawn from the study:}

$\checkmark$ Amongst all formulation, pellets prepared by solution layering on non pareils and coated with polymer showed that by varying the polymer concentration better sustained release could be achieved in non cross linkers i,e F2 have shown better drug release parameters than $\mathrm{F} 1$.

$\checkmark \quad$ Pellets coated by using crosslinking reaction shown better sustained release than normal coating.

$\checkmark$ The increase in concentration of crosslinking agent shown more sustained release from the evaluation of finished formulations.

$\checkmark \quad$ The best found formulation showed the in vitro drug release studies revealed that formulation-8(F8) shown better sustained release for $12 \mathrm{~h}$.

$\checkmark$ Best three formulation(F2,F7,F8) are subjected to accelerated stability studies shown no significant effect on the drug content and in vitro drug release from the formulation, hence it is quite stable at $40^{\circ} \mathrm{C}$ and $75 \%$ R.H. for three months.

\section{REFERENCES}

1. Abhijit Gaikwad, Parag Pathade and Late Sanjay Bidkar, Amol Pawar, Formulation and Evaluation of Multi Particulate Drug Delivery System of Glipizide by Using Crossed Linked Chitosan, Journal of Pharmacy Research 2011,4(1),300-303
2. Kammela K Chakravarthy, Mohammad Younus, Shahidulla Shaik, Sai Venkata Vedavyas Pisipati, Formulation and Evaluation of Enteric Coated Pellets of Omeprazole, International Journal of Drug Development \& Research October-December 2012 ,Vol. 4 ,Issue 4 , ISSN 0975-9344

3. Jayvadan K, Patel R, Avani F, Patel MM 2005. Formulation and evaluation of mucoadesive glipizide microspheres. AAPS Pharm.Sci.Tech. 6 (1), 10.

4. Gandhi R, Kaul C 1999. Extrusion and spheronization in the development of oral Controlled-release dosage forms. Research focus. 2(4), 160-170.

5. Palsson BO, Wheatley TA, Dressman JB 1990. Mechanism of release from pellets coated with an ethylcellulose based film, J. Cont. Release. 14, 203-213.

6. Ghebre-Sellassie I 1989. Pellets: A General Overview. GhebreSellassie, I. (Eds) Pharmaceutical Pelletization Technology, Marcel Dekker Inc. New York, 1-13.

7. Melia C, Washington N, Wilson GC 1994. Multiparticulate Controlled Release Oral Dosage Forms, Scottish Academic Press Ltd., Edinburgh, 1-12

8. Vervaet C, Remon JP 1994.Extrusion-spheronization: A literature review. Ind J Pharm. 116(1), 131-146.

9. Parikh DM, Mehta KA 2003. Extrusion/Spheronization as a granulation technique. Marcel Dekker, New York. 333-362.

10. Schmidt C, Kleinebudde P 1999. Influence of the granulation step on pellets prepared by extrusion/ Spheronization. Chem. Pharm Bull 47(3) 405-412.

11. Sinha VR, Agrawal MK, Bhinge JR 2005. Influence of formulation and excipient variables on the pellet properties prepared by extrusion-spheronization. Current drug delivery. 2,1-8.

12. Beten DB, Amighi K, Moes AJ 1995. Preparation of controlled release co- evaporates of dipyridamole by loading neutral pellets in a fluidized-bed coating system. Pharm Res. 12(9), 1269-1272.

13. Mehta A.M, Valazza M.J, Abele, S.E 1986. Evaluation of fluidized-bed processes for enteric coating systems, Pharm. Technology. 46-56.

14. Khan MA, Singh SK, Dodge J, Durrani MJ 1995. Optimization and characterization of controlled release pellets coated with an experimental latex. I: Anionic drug. Int. J. Pharm. 125(2), 243-255.

15. Umprayn K, Chitropas P, Amarekajorn S 1999. Development of terbutaline sulphate sustained release coated pellets. Drug Dev. Ind. Pharm. 25 (4), 477-491.

16. Steckel H, Nogly MF 2003. Production of chitosan pellets by extrusion /spheronization. Eur J Pharm and Biopharm. 57, 107-114.

17. Cole GC, Hogan J, Aulton M 1995. Introduction and overview of pharmaceutical coating. Pharmaceutical coating technology, Taylor and Francis Ltd. London. p. 6-7. 